\title{
Wireless sensor network node localization algorithm based on chicken swarm optimization and multi-power mobile anchor
}

\author{
Peng Chen ${ }^{1, a}$, Yongyi Mao ${ }^{2, b}$ \\ ${ }^{1}$ School of Communication and information engineering $\mathrm{Xi}^{\prime}$ an University of Posts and \\ Telecommunications, Xi' an 710061,China; \\ ${ }^{2}$ School of Electronic Engineering, \\ Xi' an University of Posts and Telecommunications, Xi' an 710061,China. \\ achenpengxupt@126.com, ${ }^{\mathrm{b}}$ maoyongyi@236.net
}

Keywords:wireless sensor network, node localization, chicken swarm optimization(CSO), multi-power mobile anchor

\begin{abstract}
In order to enhance the statistic accuracy, a new algorithm based on chicken swarm optimization(CSO) and mobile anchor was established in WSN. Firstly, the distance measurement information of unknown nodes was acquired based on different power signal, which was launched by anchor moved in target area. Then the optimal solution was estimated iteratively by different chicken swarm identities, which were identified according to fitness value.That CSO was able to improved the convergence ability of algorithms by adjusting the searching direction in space adaptively. .Overall, the results indicated that the CSO algorithm has better positioning accuracy , and could be better applied in the field of WSN localization of node random distribution.
\end{abstract}

\section{Introduction}

Recently, with the development of Internet of Things, WSN (wireless sensor network) which worked as a hot field in the inter-discipline subjects study, has already acquired more and more attention world wide. Even though WSN is widely used in target identification, collecting, calculation, tracking and controlling, the message without location information is meaningless ${ }^{[1]}$. However, different from traditional communication network, the nodes of WSN are self-organized and randomly distributed, so the energy, volume and communication distance are always limited in their working condition.

Based on the characteristics of WSN and positioning requirement, two methods named range-based and range-free ${ }^{[2]}$ are commonly used in basic positioning The former one calculated the position of the unknown nodes by trilateration or triangulation with the distance or angle between nodes. TOA, AOA, TDOA and positioning based on RSSI are common methods of range-based ${ }^{[3]}$.The latter one, instead of measuring the distance information between the nodes, works with network connectivity to estimate the location of unknown nodes. Compared with the range-based, this method is less cost and simple implemention, but lower accuracy. The common methods are APIT ${ }^{[4]}$, DV-Hop ${ }^{[5,6]}$, centroid algorithm, and Amorphous algorithm.

$L I$ et al optimize the DV-Hop positioning by artificial bee colony algorithm, which transformed the node positioning problem into the global optimization problem ${ }^{[7]}$. WAN et al constructed a triangle between an unknown node and two known anchor nodes, and calculated the distance to located the anchor position ${ }^{[8]}$.In $S H I$ 's research, a kind of convex optimization techniques with multi-power mobile anchor applied for postioning ${ }^{[9]}$. However, problems such as low accuracy and long positioning time are still exist. Therefore, a new method, which based on CSO(chicken swarm optimization) and mobile anchor by wireless communication module of sensor nodes, was established in this paper, and verified by simulation. This method based on different power signal launched by anchor in network, did not need the technique of high accuracy measuring.Every unknown node estimated self-position with received anchor signal by CSO. 


\section{Mobile model and distance estimation model}

In the target area, unknown nodes were deployed randomly in the square of $\mathrm{L} \times \mathrm{L}$ and the mobile anchor moved as $\mathrm{SCAN}^{[10]}$ route with step-size $\mathrm{s}($ Fig. 1). Along with the direction of arrow, the anchor node moved at one point in the target area, stopped and launched two levels signal of increasing power ${ }^{[1]} \mathrm{P} 1$ and $\mathrm{P} 2$, which communication ranged correspond $\mathrm{r}$ and $\mathrm{R}$. The signal included anchor coordinates and communication range. When the anchor signal received by a unknown node, it would no longer receive the higher signal at the same position. The power between a unknown node and the anchor was $\mathrm{Pi}$, if $\mathrm{Pi} \leq \mathrm{P} 1$, the estimated distance between an unknown node and the anchor was $d=r / 2$; if $\mathrm{P} 1<\mathrm{Pi} \leq \mathrm{P} 2$, the estimated distance was $d=(r+R) /$ 2 (Fig. 2).

With anchor coordinates and estimated distance $d$, the unknown node could obtain optimal solution by CSO.

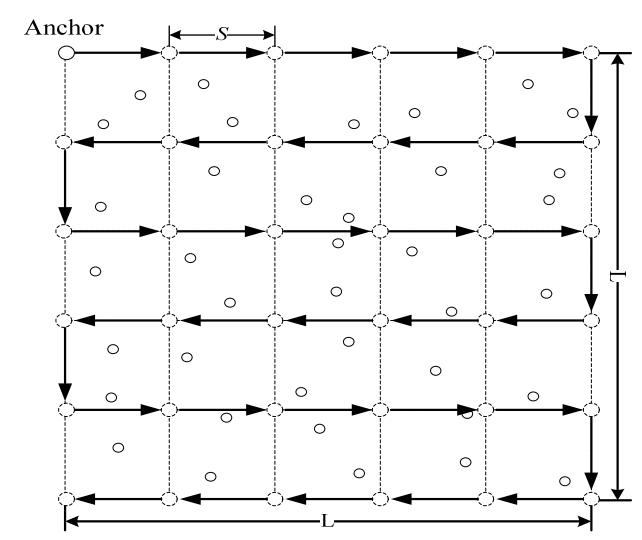

Fig.1 Mobile model

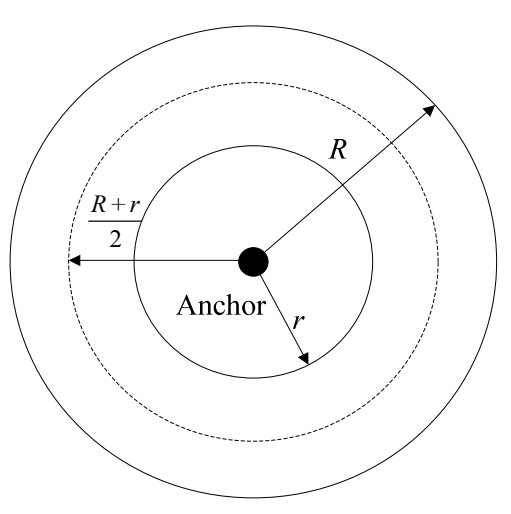

Fig.2 Distance estimation model

\section{CSO localization algorithm}

$\mathrm{CSO}$ (Chicken swarm optimization) algorithm was a new bio-inspired algorithm ${ }^{[12]}$, which simulated foraging activities of the chicken swarm by divided chickens into different subgroups. In each subgroup, every individual iterated simultaneously towards the optimal one. The mathematical description of CSO in the two-dimensional space was as follows: the population was established by $\mathrm{N}$ individuals which were potential solutions for location of unknown nodes, and the coordinates of indivaduals were $X_{i}=\left[x_{i x}, x_{i y}\right](\mathrm{i}=1,2, \ldots . . \mathrm{N})$. The optimal solution of each individual and subgroup were obtained by analysing their fitness functions, and iterating the coordinates of each individuals based on equation (1), (3), and (6).

\subsection{The four steps of CSO}

Step 1: Initialized population, defined parameters, calculated fitness value of individuals.

Step 2: Sorted individuals by fitness value, and determined identities and subgroups.

Step 3: Individuals with different identities were iterated according to different formulas , and sorted again by new fitness value every $\mathrm{G}$ time.

Step 4: Stopped iterating, and selected optimal solution from the population.

\subsection{Movement of the chickens}

The rooster with better fitness value could search for food in a wider range. The movement function of the rooster is:

$$
\begin{aligned}
& x_{i}^{t+1}=x_{i}^{t} \times\left(1+\operatorname{Randn}\left(0, \sigma^{2}\right)\right) \\
& \sigma^{2}=\left\{\begin{array}{c}
1, f_{i} \leq f_{k} \\
\exp \left(\frac{\left(f_{k}-f_{i}\right)}{\left|f_{i}\right|+\varepsilon}\right), \text { otherwise }
\end{array} k[1, N], k \neq i\right.
\end{aligned}
$$


The $x_{i}^{t}$ represented the position of the $i$ th rooster at a certain time $(\mathrm{t})$. $\operatorname{Randn}\left(0, \sigma^{2}\right)$ was a Gaussian distribution with mean 0 and standard deviation $\sigma^{2}$. The $\varepsilon$, which was the smallest constant in the computer, was used to avoid zero-division-error.$f_{k}$ was fitness value of the $k$ th rooster.

Hens followed their group-mate roosters to search for food:

$$
\begin{aligned}
& x_{i}^{t+1}=x_{i}^{t}+c_{1} \times \text { Rand } \times\left(x_{r 1}^{t}-x_{i}^{t}\right)+c_{2} \times \text { Rand } \times\left(x_{r 2}^{t}-x_{i}^{t}\right) \\
& c_{1}=\exp \left(\left(f_{i}-f_{r 1}\right) /\left(a b s\left(f_{i}\right)+\varepsilon\right)\right) \\
& c_{2}=\exp \left(\left(f_{r 2}-f_{i}\right)\right)
\end{aligned}
$$

"Rand" represented a uniform random number over [0,1]. $r 1$ represented the rooster, which was the $i$ th hen's group-mate. $r 2$ represented a random individual in the swarm. The individual moved toward better individual only if $f_{r 2}<f_{i} . c 1$ and $c 2$ were adjusted based on their actual position adaptively.

The chicks foraged with their mother. The formula is:

$$
x_{i}^{t+1}=x_{i}^{t}+F L \times\left(x_{m}^{t}-x_{i}^{t}\right)
$$

$x_{m}^{t}$ represented the position of the $i$ th chick's mother at a certain time $(\mathrm{t})$. FL represented a parameter, which kept the chick forage around its mother.

\section{3 fitness function}

Set the position of an individual in chicken swarm as $\left(\mathrm{x}^{\prime}, \mathrm{y}^{\prime}\right)$. $\mathrm{N}$ was the number of signal which an unknown node received from anchor. The coordinate of an anchor was $\left(x_{i}, y_{i}\right), i=1,2 \ldots \ldots . \mathrm{N} . d_{i}$ is the distance between a unknown node and an anchor which was calculated by the model in Fig.2. The fitness function of individual was :

$$
\text { fitness }\left(x^{\prime}, y^{\prime}\right)=\sum_{i=1}^{N}\left|\sqrt{\left(x^{\prime}-x_{i}\right)^{2}+\left(y^{\prime}-y_{i}\right)^{2}}-d_{i}\right|
$$

Fitness value represented the error between node's estimated position and real position. The smaller the fitness value was, the less the error was.

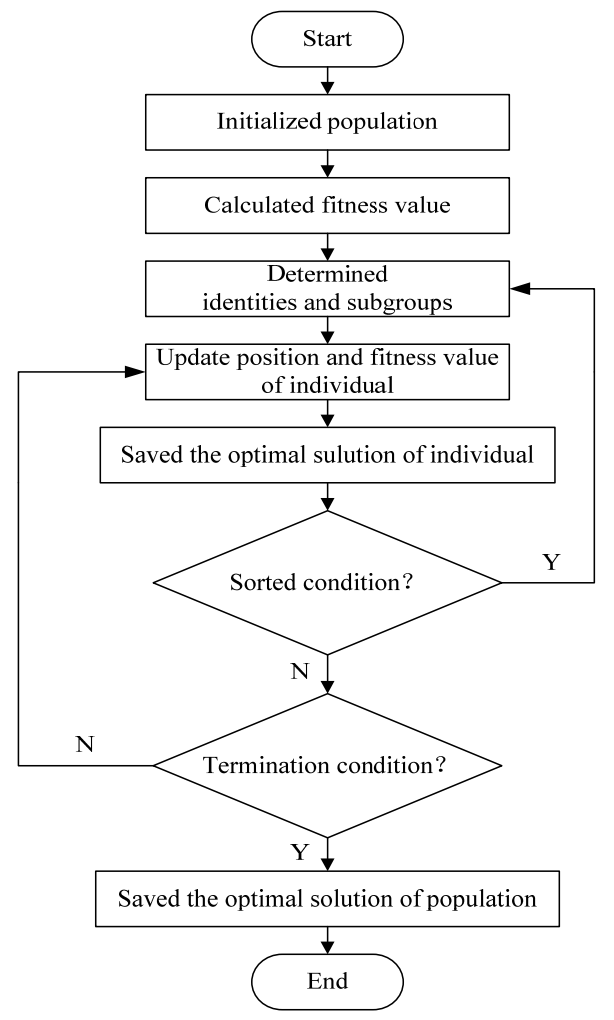

Fig.3 The flow diagram of CSO 


\section{Simulation and Analysis}

\subsection{Parameters of CSO}

One hundred sensor nodes were randomly deployed in a $100 \mathrm{~m} \times 100 \mathrm{~m}$ square simulation area. The communication range of the anchor was $15 \mathrm{~m}$ and $30 \mathrm{~m}$, the step-size was $10 \mathrm{~m}$. The population number was 40 , and the proportion of rooster, hen and chicks was $20 \%, 60 \%$ and $20 \%$, respectively. Iteration times was 20 . FL was randomly choose between 0.4 and $1^{[12]}$. The average value is obtained by 500 times randomly repeated simulation. Estimated distance between nodes and anchor was calculated by RM signal transmission model:

$$
P_{R}(d)=P_{T}-P L\left(d_{0}\right)-10 \eta \lg \left(\frac{d}{d_{0}}\right)
$$

$P_{R}(d)$ represented the power of received signal. $P_{T}$ represented emission power. $P L(d 0)$ was the signal loss on the $d 0$ meter, $\eta$ was path loss, and $d$ was the signal propagation distance.

\subsection{Performance evaluation}

The average localization error $e l_{\text {ave }}$ was used to evaluate the location result:

$$
e_{\text {ave }}=\frac{\sum_{k=1}^{N} \sqrt{\left(x_{k}-x_{k}^{\prime}\right)^{2}+\left(y_{k}-y_{k}^{\prime}\right)^{2}}}{R \times N}
$$

$\left(x_{k}, y_{k}\right)$ was the real position of an unknown node, $\left(x_{k}^{\prime}, y_{k}^{\prime}\right)$ was the estimated position, $R$ was the average communication range of mobile anchor, and $N$ was the number of unknown nodes.

\subsection{Comparison and analysis}

The line created by the real (o) and estimated $(*)$ position points of a node was the localization error (Fig. 4). Under the same condition, the error of CSO was less than that of $\mathrm{CC}^{[9]}$ and DV-hop (Fig. 4, Fig. 5, table 1). Since the DV-hop location depended on the connectivity of network, the more the nodes, the higher the accuracy. For CSO and CC methods, which located by received anchor signal, the effect of the anchor number to its location was small. Because of their less accumulated error. The CC transformed non-convex problem into convex one, which reduced the positioning accuracy. On the contrary, CSO got the location of unknown nodes with better accuracy by calculating iteratively.

In Fig.6, the step-size of the anchor is $10 \mathrm{~m}$. The location error was raised as the increase of the communication radius. Even though errors of both of these two methods were increased, the accuracy of CSO was still much better.

Table 1 Localization error comparision between CC and CSO

\begin{tabular}{cccc}
\hline Algorithm & Maximum & Minimum & Average \\
\hline CC & $13.06 \%$ & $9.76 \%$ & $11.58 \%$ \\
CSO & $11.29 \%$ & $8.57 \%$ & $9.94 \%$ \\
\hline
\end{tabular}

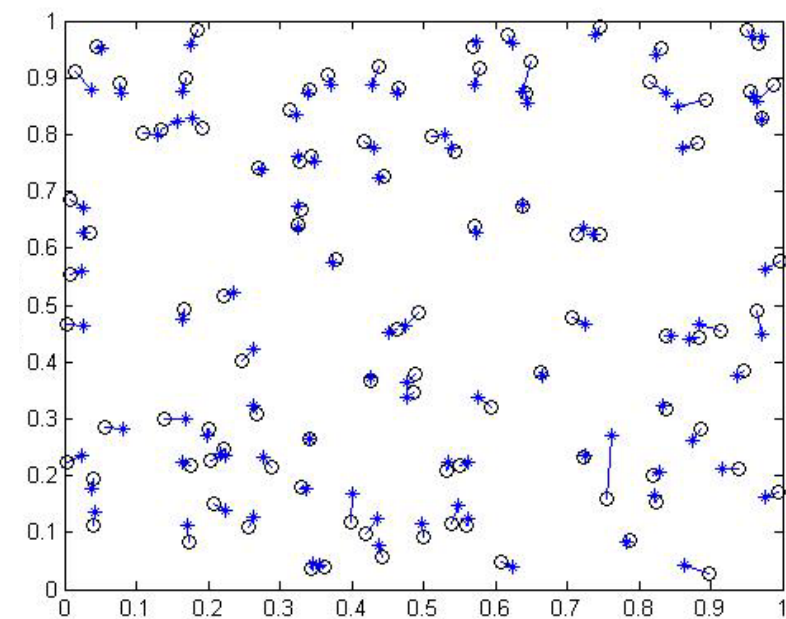

(a) $\mathrm{CC}\left(e_{\text {ave }}=12.64 \%\right)$

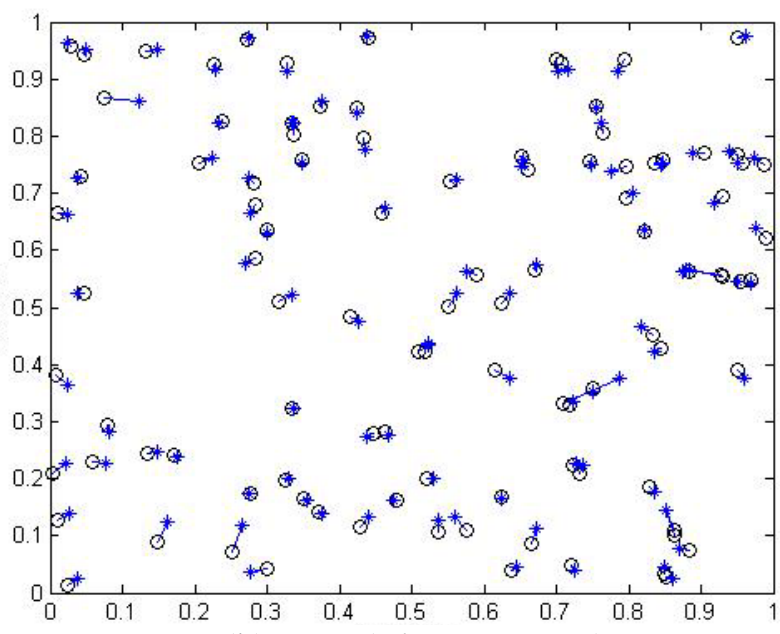

(b) $\operatorname{CSO}\left(e l_{a v e}=10.57 \%\right)$

Fig.4 CC vs. CSO under RM channel model 


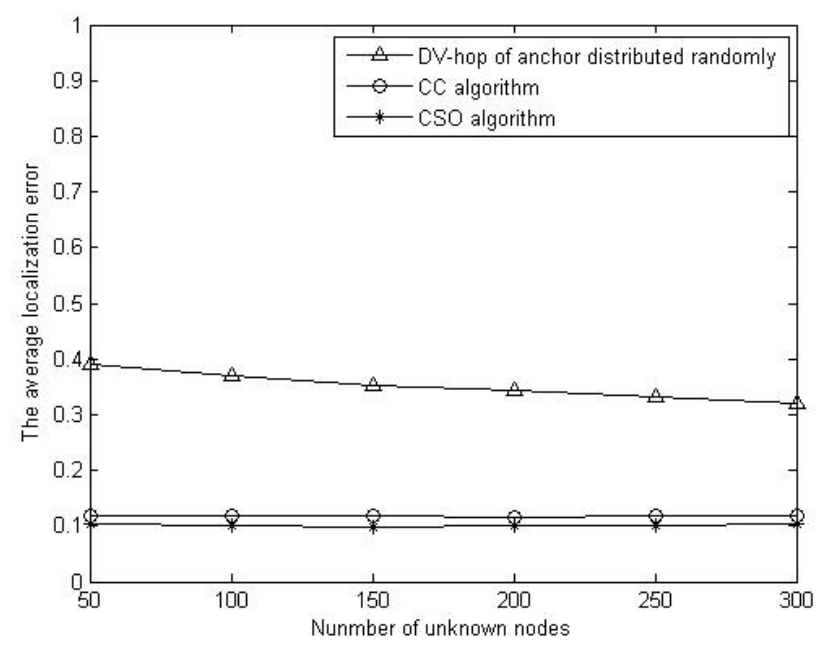

Fig.5 Number of unknown nodes vs.average localization error

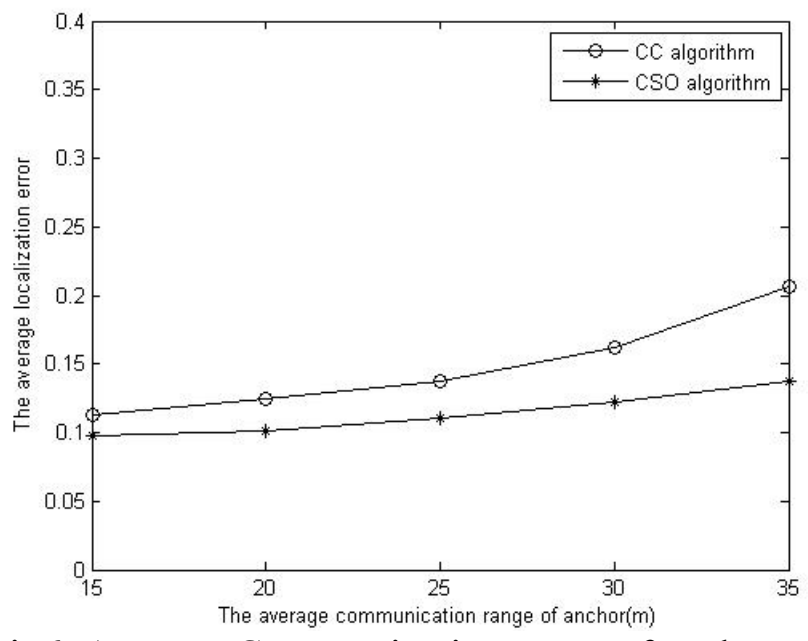

Fig6. Average Communication range of anchor vs.average localization error

\section{Conclusions}

To solve the nodes localization problems in WSN, a new algorithm with one multi-power mobile anchor and CSO algorithm was proposed, which could work without extra hardware devices. The mobile anchor moved as SCAN route, launched signal with different power, and acquired estimated distance from unknown nodes in the target area. The CSO calculated the nodes position iteratively. The simulation indicated that the localization result of CSO was more accurate than that of CC under the same condition.

\section{Acknowledgements}

This work is supported by the Natural Science Foundation of Shaanxi Province(2014JM2-6088).

\section{References}

[1] Zhu C, Wu S, Han G, et al. A tree-cluster based data gathering algorithm for industrial WSNs with a mobile sink[J]. Access IEEE, 2015, 3:381-396.

[2] Wei Q,Zhong D,Han J. Improved localisation method based on multi-hop distance unbiased estimation[J]. IET Communications, 2014, 8(16):2797-2804.

[3] Park J W, Park D H, Lee C. Angle and ranging based localization method for ad hoc network[J]. Journal of Supercomputing, 2013, 64(2):507-521.

[4] He T, Huang C, Blum B M, et al. Range-Free localization schemes for large scale sensor networks[J]. Department of Computer Science School of Engineering University Virginia, 2003:81-95.

[5] Niculescu D, Nath B. Ad hoc positioning system[J]. Globecom,2003,3:1734-1743.

[6] CHENG Chao, QIAN Zhihong, FU Caixin,et al.Genetic optimization DV-Hop localization algorithm based on error distance weighted and hop algorithm selection[J].Journal of Electronics \& Information Technology,2015(10):2418-2423.

[7] LI Mudong, XIONG Wei ,LIANG Qing. Wireless sensor networks node localization algorithm based on improved $\mathrm{ABC}$ algorithm[J]. Chinese Journal of Sensors and Actuators, 2013,26(02):241-245.

[8] WAN Guofeng, ZHONG Jun. Triangle-based localization algorithm for wireless sensor networks[J].Application Research of Computers,2013,30(1):249-251. 
[9] SHI Qingjiang, HE Chen. Multi-power level mobile beacon assisted distributed node localization algorithm[J].Journal on Communications,2009,30(10):8-13.

[10] Koutsonikolas D, Das S M, Hu Y C. Path planning of mobile landmarks for localization in wireless sensor networks[J]. Computer Communications, 2007,30:2577-2592.

[11] Du Yangyang, Mao Yongyi. Intelligent algorithm for locating nodes in wireless sensor network based on the multi-power level mobile anchor node[J].Application of Electronic Technique,2015,41(6):88-90.

[12] Meng X, Liu Y, Gao X, et al. A new bio-inspired algorithm:chicken swarm optimization[M]// Advances in Swarm Intelligence.Springer International Publishing,2014:86-94. 